\title{
THE INFLUENCES OF THE ASYMMETRIC GAIN SATURATION EFFECT ON THE DYNAMICS OF InGaAsP FABRY-PEROT LASERS
}

\author{
Veronica Dobrovolschi*, ORCID ID: 0000-0001-8361-9340, \\ Constantin Pirtac, ORCID ID: 0000-0002-7775-237X, \\ Vasile Tronciu, ORCID ID: 0000-0002-9164-2249 \\ Technical University of Moldova, 168, Stefan cel Mare Str.,MD-2004, Chisinau, Republic of Moldova \\ *Corresponding author: Veronica Dobrovolschi, veronica.dobrovolschi@fiz.utm.md
}

Received: 12. 22. 2020

Accepted: 02. 03. 2021

\begin{abstract}
This paper reports on the influence of the asymmetric gain saturation on the dynamics of InGaAsP Fabry-Perot lasers. The results are obtained by numerical simulations of multimode rate equations. The gain saturation effects that results in competition phenomena among lasing modes are investigated. A hopping multimode case is examined in detail. With means of the bifurcation analysis the regions with instabilities in the evolution of the mode intensities have been found. The following operation in the plane of two parameters are obtained: stable single mode, stable multimode, bistabile, and multimode hopping. The pulse traces of amplitudes of modes are plotted to illustrate the observed behaviors. Finally, the influence of linewidth enhancement Henry factor on the laser behavior is investigated.
\end{abstract}

Keywords: $\quad$ Mode hopping, InGaAsP lasers, multimode operation, Henry factor.

\section{Introduction}

During recent yeas InGaAsP semiconductor lasers received considerable attention due to their applications as coherent sources for optical communications. Under appropriate conditions such lasers exhibit the stable single mode operations. On the other hand, when considering saturation coefficients the laser displays several types of nonlinear effects [1 - 3]. Mode hopping is directly correlated to the noise in the total optical intensity. There are combinations of laser case temperature and injection current that lead to mode hopping and others for which the laser is stable. Recording such noise characteristics of laser diode has implications for controlling and circumventing mode hopping effects [4]. the overall intensity noise dynamics of InGaN edge emitting lasers was reported in [5]. Mode clustering usually observed in InGaN lasers enhances the mode competition and then the laser intensity fluctuation. Variations as large as $20 \mathrm{~dB}$ have been observed. The coexistence of longitudinal modes induces mode competition and then unstable bimodal lasing. This mode competition is mediated by the temporal fluctuations of spontaneous emission through the nonlinear cross saturation of the optical gain [5]. Comprehensive investigation of noise characteristics and radiation spectrum with special attention to the 
mode-hopping effect of Fabry-Pérot multiple quantum well laser diodes have been carried out [6]. Cross-correlation factor between them have been measured under stable and modehopping operation. At the mode-hopping that occurs at particular operation conditions (injection current and temperature) laser diode radiation spectrum is unstable in time [6]. A time-resolved study is presented in [7] of the single-mode and mode-switching dynamics observed in swept source vertical cavity surfing emitting lasers and swept wavelength short external cavity lasers. More complex mode-switching behaviour occurs in the external cavity laser, with the mode-hopping dynamics found to be dominated by the deterministic movement of the spectral filter.

Recently, a comparative analysis of static and dynamic measurements, on the impact of mode hopping on the overall intensity noise dynamics of InGaN edge emitting lasers is reported in [8]. The mode competition is mediated by temporal fluctuations of spontaneous emission through nonlinear cross saturation of the optical gain. In [9] a report on coherent measurements of the ultrafast dynamics, mode competition and frequency selection in a frequency-tuneable laser is presented. The used approach allows to observe hopping between lasing modes on picosecond-timescales. The physics is explained through a full multi-mode, temperature-dependent carrier and photon transport model.

This paper is concerned with investigations of the influence of asymmetric cross saturation gain on the FP laser dynamics. We report the bifurcation analysis with for the case of of multimode operation of InGaAsP semiconductor lasers. In Section 2 we introduce the setup, and an appropriate model to describe it. Section 3 presents a study of the multimode dynamics of laser and control of its operation. Finally, conclusion are given in Section 4.

\section{Laser structure and equations}

A sketch of the investigated InGaAsP laser is shown in Fig. 1. It consist of an $300 \mu \mathrm{m}$ active section pumped by an injected current $I . R_{\mathrm{f}}$ and $R_{\mathrm{b}}$ are the reflectivity of the airmaterial front facet and the outer facet of the material cavity, respectively. Front facet reflectivity $R_{\mathrm{f}}$ is $20 \%$ and back facet reflectivity $R_{\mathrm{b}}$ is $70 \%$. The width of active region is $0.3 \mu \mathrm{m}$, and the thickness is $0.1 \mu \mathrm{m}$. We consider the multimode laser operation. We assume that an injected current $I$ is injected into the active region.

The laser dynamics is analyzed using multimode rate equations of the photon number $S_{p}$ and the injected carrier number $N$

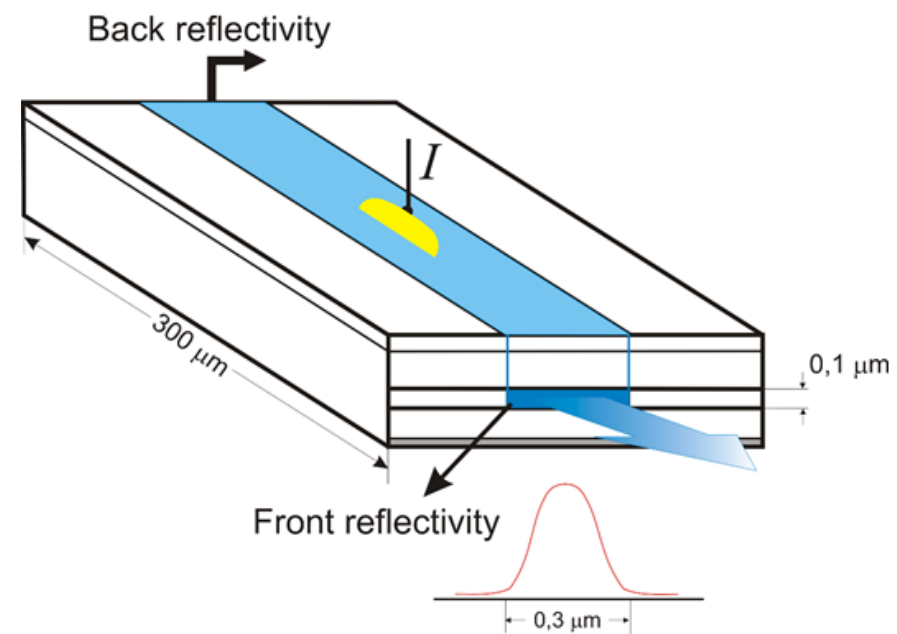

Figure 1. Setup of InGaAsP Fabry-Perot lasers.

$$
\begin{gathered}
\frac{d S_{p}}{d t}=\left(A_{p}-B S_{p}-\sum_{q} D_{p(q)} S_{q}-G_{t h}\right) S_{p}+\frac{a \xi}{V} N, \\
\frac{d N}{d t}=-\sum_{q} A_{p} S_{p}-\frac{N}{\tau_{s}}+\frac{I}{e},
\end{gathered}
$$


where $p$ is the mode number $(p= \pm 1, \pm 2 \ldots)$. We mention that in our calculations we consider 9 modes. The mode $p=0$ with wavelength $\lambda_{0}$ is the central mode. $A_{p}$ is liniar gain coefficient with the following form

$$
A_{p}=\frac{a \xi}{V}\left[\left(N-N_{g}\right)-b\left(\lambda_{p}-\lambda_{0}\right)^{2}\right]
$$

where $N_{\mathrm{g}}$ is the carrier number at the transparency. $V$ is the laser volume. $a$ is the slope of the local linear gain, and $b$ is a coefficient giving the wavelength dispersion of the linear gain. $\xi$ is the field confinement factor of the active region.

In (1) $B$ and $D$ represents the gain saturation coefficients

$$
B=B_{0}\left(N-N_{g}\right) \quad \text { and } \quad D_{p(q)}=\frac{4}{3} B+\frac{H_{c}\left(N-N_{g}\right)}{\lambda_{q}-\lambda_{p}},
$$

where, $H_{c}$ is linewidth enhancement factor [10].

The threshold gain level is given by expression

$$
G_{t h}=\frac{c}{n_{r}}\left[k+\frac{1}{2 L} \ln \frac{1}{R_{f} R_{b}}\right],
$$

where $R_{\mathrm{f}}$ and $R_{\mathrm{b}}$ are the front and back facets power reflectivity, respectively. $L$ is the laser length. $n_{\mathrm{r}}$ is the refractive index. $\mathrm{K}$ is the loss coefficient that includes internal and mirrors losses.

The following set of parameters we use in the numerical calculations $a=2.7 \cdot 10^{-12} \mathrm{~m}^{3} \mathrm{~s}^{-1}, \xi=0.2, L=2.77 \cdot 10^{-4} \mathrm{~m}, N_{g}=2.35 \cdot 10^{-8}, B_{0}=7.2 \cdot 10^{-5}, \tau_{\mathrm{s}}=2.79 \cdot 10^{-9} \mathrm{~s}$, $\lambda_{0}=785 \mathrm{~nm}, \Delta \lambda=0.31 \mathrm{~nm}$.

\section{Results and discussions}

In this section we discuss the behavior of semiconductor laser shown in Fig. 1 using the equations (1)-(5). Figure 2 illustrates the dependence of photon number $S$ on injected current calculated for nine modes after threshold current. For low current the central mode is dominant (see black line in Fig. 2).

An increase of injected current leads to mode hopping and the mode +1 (red line) become dominant. A further increase of injected current results in another hopping to mode +2 . The next hoping takes place at current $0.125 \mathrm{~mA}$ where the mode +3 is dominant. Finally, for higher currents the mode +4 is

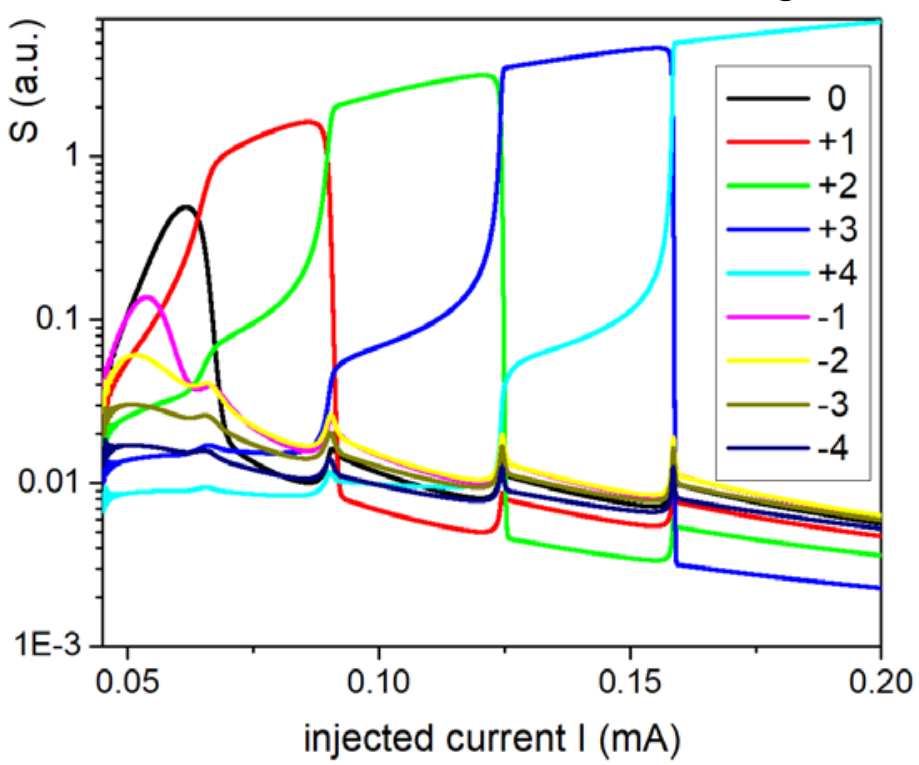

Figure 2. Photon number $S$ in the dependence of injected current $\mathrm{I}$. dominant. 
Figure 3 shows time traces of all nine modes for different regions of Figure 2. First we consider the case of currents where the mode +1 is dominant. Figure 3 a confirms by pulse traces that certainly this mode is dominant (red line). One can see that in this case the injected current is chosen just after hoping since the mode 0 is located in the near vicinity to mode +1 . Figure $3 \mathrm{~b}$ shows other hoping in time between mode +2 to +3 . After 3 ns the mode +3 become dominant and one can see tin this case the single mode operation since the other modes has the lower photon number S. Finally, Figure $3 c$ shows a strong sinle mode operation of mode +4 for high injected currents.
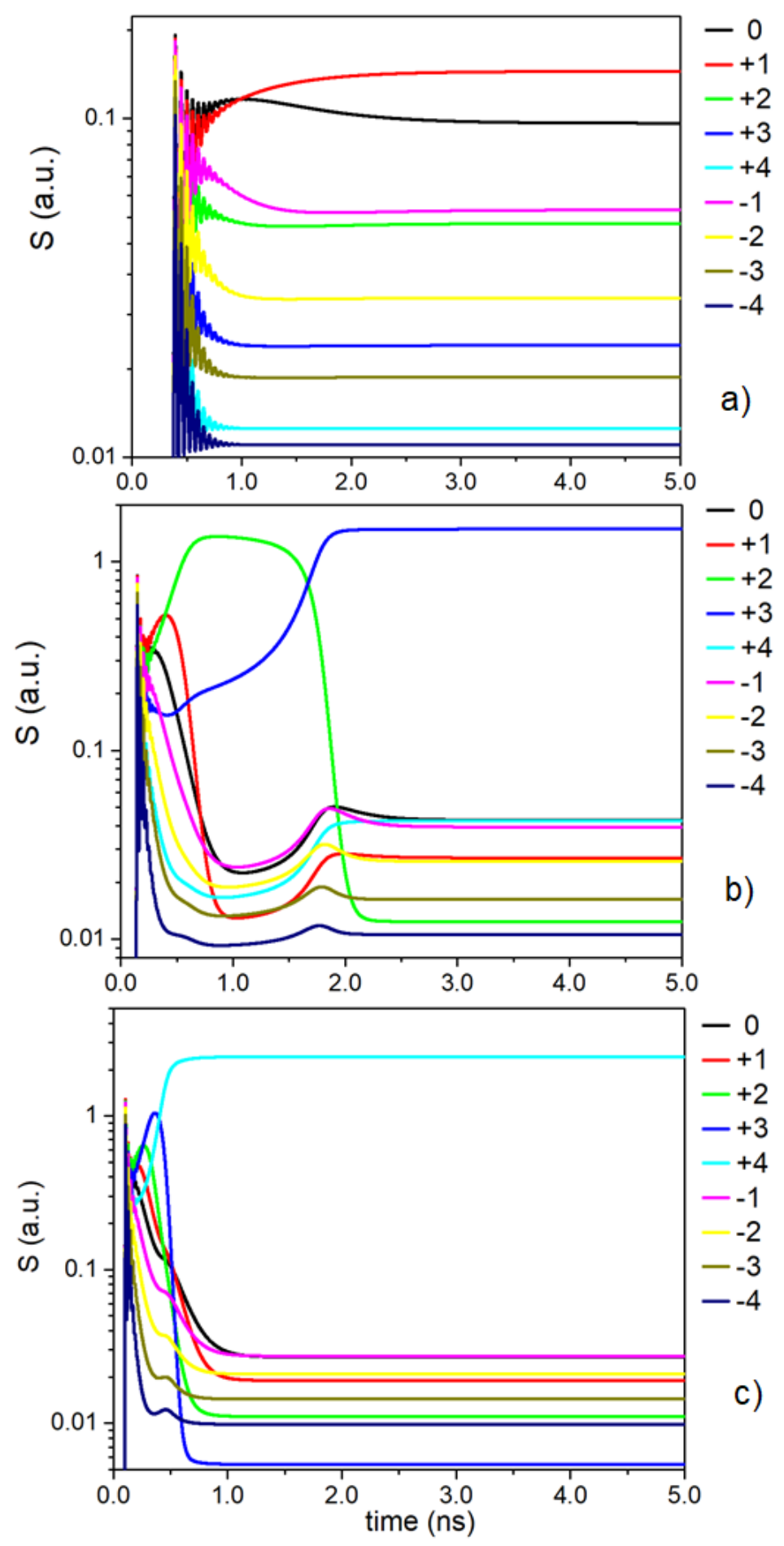

Figure 3. The time evolution of total output number $S$. 
In what follows we show in Figure 4 the classifications of possible states of laser operations in the plane of two parameters $H_{\mathrm{c}}-l / I_{\text {th }}$. For small values of parameter $H \mathrm{c}$ the single mode dominates the operation of laser. When $\mathrm{Hc}$ is increased the bistable phenomena is present in the laser.The bistability is realized between modes $+1,+2$ and +3 . For higher $\mathrm{Hc}$ the laser shows two regions multimode hoping. Finally, for both higher values of injected current and $\mathrm{Hc}$ the single mode of mode +4 is observed. This is confirmed also by Figure 3c.

Figure 5 shows multimode operation of laser that correspond to region of "multimode CW" of Fig. 4. The figure indicates that the modes display a quasiperiodic evolution in time. There is no any dominant modes. We only mention that, the photon number of some modes $(0,+1,+2+3)$ are higher that of others.

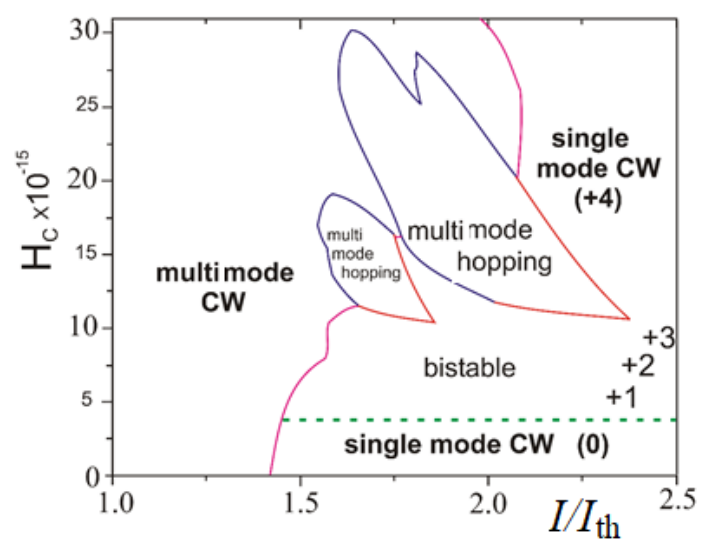

Figure 4. Regions of different laser behavior in the plane of two parameters.

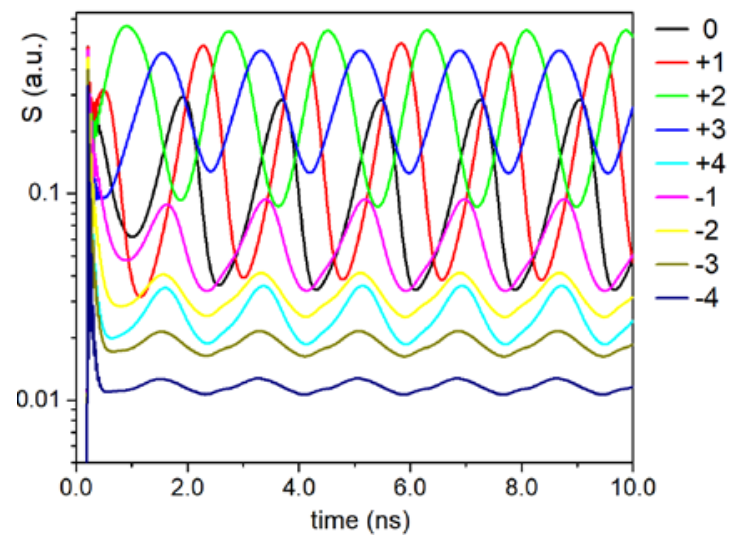

Figure 5. Multimode operation.

\section{Conclusions}

We have studied the mode hopping dynamics of multimode InGaAsP Fabry-Perot lasers composed by a single cavity. The laser operation is classified into stable single mode, stable multimode, hopping multimode and bistable. We have shown that the dynamics of such lasers strongly depend on asymmetric cross saturation gain. Operation of InGaAsP lasers at high currents is characterized by rotation of the lasing mode among several long wavelength modes. Finally, we found that the InGaAsP lasers exhibit stable single mode operation under high currents. We believe that our work provides a good basis for future study and, in particular, provides some pointers for more detailed investigations of dynamics of multimode InGaAsP lasers and their control in different applications.

Acknowledgments. This work was supported by project 20.80009.5007.08 "Study of optoelectronic structures and thermoelectric devices with high efficiency".

\section{References}

1. Yamada M. Theoretical analysis of nonlinear optical phenomena taking into account the beating vibration of the electron density in semiconductor lasers. In: Journal of Applied Physics, 66, 1989, pp. 81-89.

2. Ahmed M., Yamada, M. Influence of instantaneous mode competition on the dynamics of semiconductor lasers. In: IEEE Journal of quantum electronics, 38 (6), 2002, pp. $682-693$.

3. Ahmed M. Numerical characterization of intensity and frequency fluctuations associated with mode hopping and single-mode jittering in semiconductor lasers. In: Physica D, 176, 2003, pp. 212-236.

4. Acsente T. Laser diode intensity noise induced by mode hopping. In: Romanian Reports in Physics, 59 (1), 2007, pp. 87-92. 
5. Congar A., Hussain K., Pareige C., Butte R., Grandjean N., Besnard P., Trbaol S. Impact of mode-hopping noise on InGaN edge emitting laser relative intensity noise properties. In: IEEE Journal of quantum electronics, 54(1), 2008, pp. 1-7.

6. Pralgauskaite S., Palenskis V., Matukas J., Saulys B. Analysis of mode-hopping effect in Fabry-Pérot multiple-quantum well laser diodes via low frequency noise investigation. In: Solid-State Electronics, 79, 2013, pp. 104-110.

7. Butler T.P., Goulding D., Kelleher O, Shaughnessy B., Slepneva S., Hegarty S.P., Huet G. Direct experimental measurement of single-mode and mode-hopping dynamics in frequency swept lasers. In: Optics Express, 25 (22), 2017, pp. 27464-27474.

8. Congar A. et al., Impact of Mode-Hopping Noise on InGaN Edge Emitting Laser Relative Intensity Noise Properties, IEEE Journal of Quantum Electronics, 54 (1), 2018 pp. 1-7. doi: 10.1109/JQE.2017.2774358

9. Kundu I. et al Ultrafast switch-on dynamics of frequency-tuneable semiconductor lasers. Nature Communication 9, 2018, p 3076, doi.org/10.1038/s41467-018-05601-x

10. Henry C. Phase noise in injection lasers. In: Journal lightwave technology, 4 (3), 1986, pp. 298-311. 